5. Eyal, N., Lipsitch, M. \& Smith, P. G. J. Infect. Dis. 221, 1752-1756 (2020).

6. van Doremalen, N. et al. Nature 586, 578-582 (2020).

7. Yu, J. et al. Science 369, 806-811 (2020).

8. Eichner, M., Schwehm, M., Eichner, L. \& Gerlier, L. BMC Infect. Dis. 17, 308 (2017).
Acknowledgements

The research reported in this paper was supported by US National Institutes of Health National Institute of General Medical Sciences grant 1R01 GM12428003S1 (supplement); National Science Foundation grant 2032084; and National Institute of Allergy and
Infectious Diseases Centers of Excellence for Influenza Research and Surveillance (CEIRS) grant HHSN272201400004C.

Competing interests

The authors declare no competing interests.

\title{
Toward superhuman SARS-CoV-2 immunity?
}

To the Editor - If asked, many scientists would probably agree with the statement 'Natural infection gives better immunity than vaccination'. Indeed, if one survives the infection, there are certainly many pathogens for which natural infection induces stronger immune responses and more long-lived immunity than does vaccination. Measles is prototypic of this ${ }^{1}$. While there was a clear risk, after infection, of death, encephalitis and pneumonia before there was a vaccine, survivors gained lifelong immunity. Vaccination against measles, on the other hand, requires two shots and may not offer lifelong complete protection but has proven to be good enough to keep the disease in check when widely implemented.

In contrast to the measles virus, there are a number of pathogens for which vaccination generates stronger immune responses and more-effective protection against disease than does natural infection. In these cases, the man-made vaccine is 'superhuman'; that is, it gives humans immune responses superior to those generated in response to infection. The bacterium that causes tetanus is a notable example of this. Infection with this pathogen results in production of the highly potent tetanus toxin in small amounts that are sufficient to cause severe disease but not enough to generate a strong immune, particularly antibody, response. On the other hand, vaccination with an inactivated form of the toxin (tetanus toxoid) generates antibody responses sufficient to provide protection against the toxin for a decade and probably longer ${ }^{2}$. Hence, vaccination is recommended even for those who have been infected with the bacterium that causes tetanus and have shown clinical symptoms, as well as those who have been merely potentially exposed.

Another example from the bacterial world is Haemophilus influenza type b (Hib). Hib causes a variety of serious conditions, including meningitis, pneumonia and septicemia. The surface of the bacterium is protected by a coating of sugars, which typically induce rather poor antibody responses. However, the responses can be greatly enhanced by linkage of the sugars to a protein in a vaccine in preparations known as 'glycoconjugates'3. The typical responses to vaccination are therefore greatly enhanced relative to the responses to natural infection. The vaccine is now given to children under the age of 2 years in many developed countries in particular and has greatly reduced the incidence of meningitis due to Hib.

Among viruses, two classic cases in which vaccines generate immunity superior to that generated by natural infection are varicella zoster virus, which can lead to shingles, and human papillomavirus (HPV), some strains of which cause various malignancies, including cervical, penile and oropharyngeal cancer. Varicella zoster virus typically causes chickenpox in children and young adults and is resolved but rendered latent so that when re-activated in later life, it can lead to shingles. Immunity arising from the primary infection does not prevent the disease in those who develop shingles. However, the recently developed vaccines Zostavax and Shingrix do offer protection against shingles. Shingrix protects around $90 \%$ vaccinees across all age groups, and it is suggested for an extended time period ${ }^{4}$. Protection seems to be antibody based but with important contributions from $\mathrm{CD} 4^{+} \mathrm{T}$ cells.

The quintessential example of immunity superior to that induced by infection is the vaccine against HPV. The HPV strains that cause genital cancers enter the body via genital mucosal surfaces, and the antibody responses induced are low and take a long time to develop-more than 8 months in one study ${ }^{5}$. In contrast, two or three sequential intramuscular injections of one of the vaccines against HPV induce potent neutralizing antibody responses that have been shown directly in an animal model to prevent entry of the virus into target cells and the establishment of infection ${ }^{6}$. The vaccines against HPV are based on the incorporation of a single viral surface protein into virus-like particles. They have been shown to offer complete protection against cervical cancer.

Where does the coronavirus SARS-CoV-2 lie along the spectrum of natural infection versus vaccine-induced protective efficacy? The answer to this question will be known only as more data are collected from ongoing natural infection and vaccine studies; the initial results from interim analyses by Pfizer/ BioNTech and Moderna of mRNA vaccines against SAR-CoV-2 showing a reduction in infections of around $95 \%$ are very encouraging ${ }^{7}$. There are a number of other promising signs for vaccines. Protection against infection and disease has been associated with neutralizing antibodies in both vaccine studies and passive-antibody-transfer studies in animal models ${ }^{8}$. Furthermore, passive antibodies seem to have beneficial effects on established early SARS-CoV-2 infection in humans, which suggests that they can contribute to protection ${ }^{9}$. Many of the current vaccines in clinical trials ${ }^{10}$ induce high levels of neutralizing antibodies that animal model studies predict would provide protection. Furthermore, even if the levels reached do not provide complete sterilizing immunity and are insufficient to prevent the upper-respiratory-tract symptoms typical of the common cold, they may prevent serious lower-respiratory-tract disease. The disadvantage of such an outcome is that the vaccine probably would not prevent ongoing transmission from an infected vaccinee. In contrast to many of the vaccines, natural infection induces highly variable levels of neutralizing antibodies, a proportion of which may not provide immunity. At the patient level, there are isolated reports of re-infection with SARS-CoV-2 associated with an insufficient initial antibody response. A second likely contributor to protection against SARS-CoV-2 is cellular immunity ${ }^{11}$, although the data on its importance are not yet clear. A number of vaccines are expected to induce substantial cellular immune responses. One important unknown factor in the context of both natural infection and vaccination is the durability of immune responses. Multiple longitudinal cohort studies of antibody levels after COVID-19 have shown that they are variable, with some showing durability over several months and others showing some 'fall-off'. The durability of antibody 
responses is likely to be 'tweakable' through judicious choice of vaccines. In general, extensive molecular studies of SARS-CoV-2 and neutralizing antibody responses will be of value should rational design strategies be needed to generate optimal vaccines ${ }^{12}$.

Overall, we are optimistic, given the number of platforms being investigated and the huge ongoing efforts, that a vaccine (or vaccines) against COVID-19 with immune responses and protection superior to that achieved through natural infection is an achievable goal.

\section{Dennis R. Burton (D) $1,2 \bowtie$ and}

Eric J. Topol(D) 1,3凶

${ }^{1}$ The Scripps Research Institute, La Jolla, CA, USA.

${ }^{2}$ Ragon Institute of MGH, Harvard and MIT,
Cambridge, MA, USA. ${ }^{3}$ Scripps Translational

Research Institute, La Jolla, CA, USA.

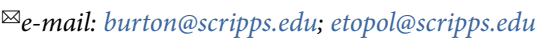

Published online: 30 November 2020

https://doi.org/10.1038/s41591-020-01180-x

References

1. Amanna, I. J. \& Slifka, M. K. Curr. Top. Microbiol. Immunol. 428, $1-30(2020)$.

2. Hammarlund, E. et al. Clin. Infect. Dis. 62, 1111-1118 (2016).

3. Rappuoli, R., De Gregorio, E. \& Costantino, P. Proc. Natl Acad. Sci. USA 116, 14-16 (2019).

$\neg$ 4. Cunningham, A. L. et al. J. Infect. Dis. 217, 1750-1760 (2018).

5. Carter, J. J. et al. J. Infect. Dis. 174, 927-936 (1996).

6. Day, P. M. et al. Cell Host Microbe 8, 260-270 (2010).

7. Cohen, J. Science https://www.sciencemag.org/news/2020/11/

just-beautiful-another-covid-19-vaccine-newcomer-moderna-suc ceeds-large-scale-trial (2020).

8. Klasse, P. J., Nixon, D. F. \& Moore, J. P. Preprints 2020 2020090166 (2020).
9. DeFrancesco, L. Nat. Biotechnol. 38, 1242-1252 (2020).

0. Krammer, F. Nature 586, 516-527 (2020).

11. Lipsitch, M., Grad, Y. H., Sette, A. \& Crotty, S. Nat. Rev. Immunol. 20, 709-713 (2020).

12. Burton, D. R. \& Walker, L. M. Cell Host Microbe 27, 695-698 (2020).

Acknowledgements

D.R.B. receives financial support from The National Institute of Allergy and Infectious Diseases, the Bill \& Melinda Gates Foundation, Ragon Institute, The International AIDS Vaccine Initiative and the Pendleton Trust. Funding for E.J.T. was provided by the Clinical and Translational Science Award (CTSA grant UL1TR002550) from the National Center for Advancing Translational Sciences of the US National Institutes of Health.

D.R.B. and E.J.T. conceived of the idea and wrote the piece together.

\section{Automated screening of COVID-19 preprints: can we help authors to improve transparency and reproducibility?}

To the Editor-The COVID-19 pandemic has thrust preprints into the spotlight, attracting attention from the media and the public, as well as from scientists. Preprints are articles not yet published in a peer-reviewed journal, and as such they offer a unique opportunity to improve reporting. The Automated Screening Working Group (https://scicrunch.org/ ASWG/about/COVIDPreprint) aims to provide rapid feedback that may help authors of COVID-19 preprints to improve their transparency and reproducibility.

One quarter of COVID-19 papers published have been preprints. Most of these appear on medRxiv; others appear on bioRxiv or other servers ${ }^{1}$. Although publishing results in preprints allows them to be posted rapidly, the absence of traditional peer review has raised concerns about preprint quality. Unfortunately, it has been impossible for scientists to keep pace with the thousands of COVID-19 preprints published since February. Preprints are vetted before posting to confirm that they describe scientific studies and to prevent posting on topics that could damage public health; however, routine assessment of manuscript quality or flagging of common reporting problems is not feasible at this scale.

Although automated screening is not a replacement for peer review, automated tools can identify common problems. Examples include failure to state whether experiments were blinded or randomized ${ }^{2}$, failure to report the sex of participants ${ }^{2}$ and misuse of bar graphs to display continuous data ${ }^{3}$. We have been using six tools ${ }^{4-8}$ to screen all new medRxiv and bioRxiv COVID19 preprints (Table 1 ). New preprints are screened daily ${ }^{9}$. By this means, reports on more than 8,000 COVID preprints have been shared using the web annotation tool hypothes.is (RRID:SCR_000430) and have been tweeted out via @SciScoreReports (https://hypothes.is/users/sciscore). Readers can access these reports in two ways. The first option is to find the link to the report in the @SciScoreReports tweet in the preprint's Twitter feed, located in the metrics tab. The second option is to download the hypothes. is bookmarklet. In addition, readers and authors can reply to the reports, which also contain information on solutions.

Screening of 6,570 medRxiv and bioRxiv COVID-19 preprints posted before 19 July revealed several interesting results. $13.6 \%$ of preprints shared open data and $14.3 \%$ shared open code, making it easier for others to reuse data or reproduce results. Approximately one third (34.4\%) of COVID-19 preprints acknowledged at least one study limitation. $7.3 \%$ of preprints included bar graphs of continuous data.
This is problematic because many different datasets can lead to the same bar graph, and the actual data may suggest different conclusions from those implied by the summary statistics alone ${ }^{3}$. Therefore, authors should use dot plots, box plots or violin plots instead ${ }^{3}$. Among papers with color maps, $7.6 \%$ used rainbow colormaps, which are not colorblind safe and also create visual artifacts for viewers with normal vision ${ }^{7}$. Rainbow color maps should be replaced with more-informative color maps that are perceptually uniform and colorblind accessible, such as viridis ${ }^{7} .1,775$ preprints (27\%) contained an ethics approval statement for human or animal research. This suggests that nearly three quarters of COVID-19 preprints are secondary or tertiary analyses, modeling studies or cell line studies that do not require approval. Although there are known sex differences in COVID $-19^{10}$, only $20 \%$ of all COVID-19 preprints, and $38 \%$ of preprints with an ethics approval statement, address sex as a biological variable. Statements regarding sample size calculations (1.4\%), blinding $(2.7 \%)$ and randomization (11.4\%) were uncommon, even among studies that contained a human ethics statement (present in $2.4 \%, 5.4 \%$ and $12.6 \%$, respectively). Many COVID-19 preprints are modeling studies, however, and hence these criteria 